\title{
Modelos de acceso abierto en educación y ciencia
}

\author{
Karen Isabel Cabrera-Peña
}

Universidad del Rosario, Colombia

kicabrerap@gmail.com

\section{Resumen}

Este artículo presenta los modelos de acceso abierto en educación y ciencia, implementados en seis paises pioneros en el tema (Argentina, Australia, Brasil, Estados Unidos, Inglaterra y España), desde la perspectiva de la Unión Europea.

El análisis parte del marco politico y legal de acceso abierto, hasta la interrelación de los modelos implementados en cada uno de los países de estudio. Se concluye que lo "abierto" contribuye a la calidad de los procesos de información y conocimiento; sin embargo, existen dificultades tecnológicas y legales para su implementación.

\section{Palabras clave}

Política educacional, educación superior, educación comparada, modelos de enseñanza, acceso abierto. (Fuente: Tesauro de la Unesco).

Recepción: 2013-01-30 / Envío a pares: 2013-04-10 / Aceptación por pares: 2014-06-28 / Aprobación: 2014-07-18 DOI: 10.5294/edu.2014.17.2.7

Para citar este artículo / To reference this article / Para citar este artigo

Cabrera-Peña, K. I. (2014). Modelos de acceso abierto en educación y ciencia. Educ. Educ. 17 (2), 321-338. Doi. 10.5294/edu.2014.17.2.7 


\title{
Open Access Models in Education and Science
}

\begin{abstract}
The article outlines the open access models in education and science being implemented in six countries that are pioneers in the field (Argentina, Australia, Brazil, England, Spain and the United States), from the perspective of the European Union. The analysis starts with the political and legal framework of open access, then proceeds to consider the association or connection between the models implemented in each of the countries under study. The conclusion is that the "open" model contributes to the quality of processes for information and knowledge; however, there are technological and legal difficulties in its implementation.
\end{abstract}

\section{Key words}

Educational policy, higher education, comparative education, teaching models, open access. (Source: Unesco Thesaurus). 


\title{
Modelos de acesso aberto em educação e ciência
}

\author{
Resumo
}

Este artigo apresenta os modelos de acesso aberto em educação e ciência, implantados em seis países pioneiros no tema (Argentina, Austrália, Brasil, Estados Unidos, Inglaterra e Espanha), sob a perspectiva da União Europeia.

A análise parte do marco politico e legal de acesso aberto, até a inter-relação dos modelos implantados em cada um dos países de estudo. Conclui-se que o "aberto" contribui para a qualidade dos processos de informação e conhecimento; contudo, existem dificuldades tecnológicas e legais para sua implantação.

\section{Palavras-chave}

Política educacional, educação superior, educação comparada, modelos de ensino, acesso aberto. (Fonte: Tesauro da Unesco). 


\section{Introducción}

El acceso a las fuentes de información científica y cultural es el principal proceso para la generación de nuevos conocimientos, convirtiéndose en un factor clave en el desarrollo de la sociedad del siglo $X X I$. Cuando se permite el acceso al conocimiento se disminuyen las brechas económicas, políticas y culturales entre las comunidades (Arencibia, 2006).

La difusión y publicación de los avances científicos y académicos han cambiado: hace algunos años la única manera eficiente de difundir la información era a través de libros y publicaciones periódicas; ahora, con el desarrollo y la expansión tanto de la Internet como de las tecnologías de la información, se crearon nuevas formas de divulgación y, por tanto, innovadoras fórmulas para acceder al conocimiento (Parada, 2005).

En este contexto nace el concepto de "acceso abierto" empleado para permitir el acceso libre y gratuito a lectores a través de versiones digitales online de artículos científicos, bajo la premisa de que la información puede reutilizarse siempre que se haga un uso legítimo y no comercial de la obra y se reconozca la autoría de los creadores (Parada, 2005). Posteriormente, esta iniciativa se amplió para recursos educativos abiertos, datos de gobierno abierto y políticas de acceso a galerías, bibliotecas, archivos y museos.

Como primer precedente se encuentra "La iniciativa de acceso abierto" firmada en Budapest que lo define como la

disponibilidad gratuita en la Internet pública para que cualquier usuario pueda leer, descargar, copiar, distribuir e imprimir con la posibilidad de buscar o enlazar todos los textos de artículos [...] o utilizarlos para cualquiera otro propósito legal sin barreras financieras, legales o técnicas distintas de la fundamental de ganar acceso a la propia Internet (Iniciativa de Acceso abierto, 2002).
Más adelante, en el año 2003, esta iniciativa fue ratificada por las Declaraciones de Bethesda y Berlín?.

Paralelo a estas declaraciones, varios países han desarrollado iniciativas de acceso abierto a la información científica y académica por medio de herramientas legales, como propuestas legislativas y ejecución de políticas públicas. Particularmente Australia y Estados Unidos han concretado sistemas fuertemente consolidados de acceso abierto no solo en áreas como las ciencias, también en cultura y educación.

Parte de la justificación de la implementación de estas políticas se ha visto respaldada por estudios e informes tales como:

- Scientific Publications. Free for all?: estudio solicitado por el Congreso de Inglaterra en el año 2004, se mostró cómo las editoriales estaban añadiendo costos al proceso de producción y difusión de la ciencia y no eran transparentes con ello (House of Commons, 2004).

- Venturous Australia del Gobierno federal australiano sobre el Sistema Nacional de Innovación: el informe señala la obligación de los gobiernos australianos de adoptar estándares internacionales de publicación abierta, también anuncia que el material divulgado para información pública debe ser publicado bajo licencias Creative Commons (Departamento de Industria, s.f.).

- El mercado de libros técnicos y científicos en Brasil. Subsidio público y acceso al conocimiento: el reporte realizado en el año 2008 presenta la estrecha relación entre la bibliografía de los estudios universitarios y la financiación pública, lo que obliga a pensar en el impacto que tiene el acceso en el conocimiento (Craveiro, Machado y Ortellado, 2008).

- Engage, getting on with Govenment 2.0: el informe de 2009 resalta la necesidad de contar

$1 \quad$ Firmadas el 11 de abril y el 6 de octubre respectivamente. 
con información pública abierta, accesible y reutilizable. Anima al gobierno a enfrentar los temas de derechos de autor adoptando licencias Creative Commons estándar (Comisión de información de Australia, 2009).

- Open Data Study: en 2010 la Open Society Foundation (OSF) publicó un estudio sobre la importancia de la transparencia en el rendimiento de las administraciones. Identificó el modelo óptimo detrás de las experiencias open data de EE.UU.y del Reino Unido que pueda ser replicable en países de ingresos medios y en desarrollo (Hogge, 2010).

- Informe Hargreave: realizado por el profesor Ian Hargreaves del Centro de Estudios Digitales de Economía Digital de la Universidad Cardiff en el Reino Unido. Propone una reforma integral al sistema de propiedad intelectual resaltando que si la ley no se modifica para ser más flexible ante los constantes cambios tecnológicos, se convertirá en un obstáculo real para el desarrollo económico de Inglaterra; de hecho afirma que ya lo es para los países en desarrollo (Hargreaves, 2011).

- $\quad$ El desafío hacia el gobierno abierto en la hora de la igualdad: presentado por la Comisión Económica para América Latina y el Caribe (Cepal). Entre otros aspectos reflexiona y propone la apertura de datos para los países de la región (Concha y Naser, 2012).

- Accessibility, sustainability, excellence: how to expand access to research publications: fue presentado en el 2012 por el gobierno de Inglaterra con una serie de recomendaciones para mejorar el acceso a los resultados de investigación.

- Celebración de la década de existencia del concepto acuñado por Unesco en el Global OER Congress: en junio de 2012 se presentó la encuesta de políticas gubernamentales de recursos educativos abiertos que recoge las experiencias reportadas por varios países en el mundo. Estos datos y resultados exhiben un listado de informes que han sido encargados por los gobiernos de los diferentes países. Entre ellos se destaca el estudio encargado al Ministerio de Educación Nacional de Colombia sobre el estado del arte nacional e internacional en acceso abierto (2010/2011) y sobre recursos educativos digitales en el país (Hoosen, 2012).

- Informe de la consulta latinoamericana y del Caribe sobre acceso abierto a información e investigación científica: el informe de la consulta realizada en Kingston durante marzo de 2013 presenta una serie de estrategias, en su mayoría de concientización e implementación de políticas públicas, para lograr mejor y mayor acceso abierto a información en Latinoamérica y el Caribe (Unesco, 2013).

\section{Marco legal y político del acceso abierto}

Teniendo en cuenta los rápidos y constantes cambios en los últimos años de las Tecnologías de la Información y las Comunicaciones (TIC) se han desplegado innovadoras formas de desarrollar, de manera eficiente y dinámica, el procesamiento de investigación que permite el acceso universal a la producción intelectual. Esta nueva dinámica de "compartir" ha generado interesantes proyectos que propenden por la cultura, la educación y la ciencia como aspectos fundamentales para el crecimiento económico y social que finalmente deben ser respaldados y asegurados por los Estados democráticos (Arencibia, 2006).

En este orden de ideas, los Estados han suscrito acuerdos internacionales que pretenden generar compromisos, como la Declaración de Principios de la Cumbre Mundial sobre la Sociedad de la información del año 2003 que afirma el deseo de "construir una sociedad de la información centrada en la persona, integradora y orientada al desarrollo, en que todos puedan crear, consultar, utilizar y compartir la información y el conocimiento", reconociendo que 
"gran parte de los elementos constitutivos de esta sociedad son el fruto de los avances científicos y técnicos que han sido posibles gracias a la comunicación mutua de los resultados de la investigación" (Declaración de Principios de la Cumbre Mundial sobre la Sociedad de la información, 2003).

Si bien se reconoce la importancia de la incorporación de instrumentos políticos y legales que permitan el acceso, lo cierto es que existen algunas limitaciones que no facilitan este ejercicio. En el caso de la propiedad industrial hay empresas privadas titulares de patentes que pueden restringir el conocimiento de fórmulas y esquemas de invención por un tiempo determinado (Landes y Posner, 2003), y en el derecho de autor existen condiciones para conocer y compartir información pues se necesita una autorización del titular de los derechos, la plena identificación del autor para reconocer la obra y en algunos casos pagar por ella (Plata, 2010). Lo anterior indica que la mayoría de los recursos a los que accedemos digitalmente están sujetos a derechos y obligaciones, tanto del titular como del usuario, y cuando existen barreras legales muy restrictivas que buscan proteger a algunas de las partes se quebranta la posibilidad de acceder a contenidos bajo el concepto de abierto.

Es por esta razón que las administraciones públicas se han visto en la necesidad de trabajar en proyectos de tipo económico, legal y tecnológico que permitan mayor acceso al conocimiento a través de políticas que delimiten marcos jurídicos seguros en el control de los contenidos a los que eventualmente pueden accederse digitalmente (Iglesias y Labastida, 2006). En este contexto "abierto" muestra la posibilidad de utilizar recursos digitales tales como datos, resultados de producción científica, material educativo o manifestaciones culturales sin pago alguno por instrumentos tecnológicos ${ }^{2}$ que adicionalmente también cuentan con los permisos o las características legales que consienten su publicación y circulación en la red (Arencibia, 2006).

2 Interoperables y en formatos estándar que varían según los requerimientos que se van construyendo o desarrollando para cada tipo de recursos.
Para materializar las políticas públicas de acceso abierto, los Estados han partido de la premisa que para difundir recursos de forma flexible es necesario gestionar previamente el derecho de autor de los contenidos que circulan, esto implica generar autorizaciones para negociar los derechos patrimoniales de las obras como la reproducción, comunicación, distribución y modificación con la finalidad de evitar responsabilidad por infracciones a estos derechos. Las autorizaciones son generales respecto a cada proyecto de acceso abierto dado que negociar la autorización de derechos de autor obra por obra consumiría gran cantidad de tiempo y dinero (Iglesias y Labastida, 2006).

Particularmente estas autorizaciones son para aquellos casos en que el modelo de negocio o sustentabilidad económica no depende de la transacción o el permiso de uso del autor, como sucede con la mayoría de las producciones intelectuales financiadas con dinero público, en donde la remuneración del autor es precisamente el crédito que le hace el Estado y, por tanto, si se suman esas dos características (dinero público que financia la obra y remuneración que no depende del control de la misma) resulta apenas lógico pensar en un entorno favorable al acceso abierto.

Algunas veces por el paso del tiempo o por las limitaciones y excepciones expresadas en la ley el control del titular sobre la obra se reduce y esta se convierte de dominio público. Cuando una obra es de dominio público cualquier persona puede acceder a ella sin ninguna autorización, es así como el permiso de gestión de derechos patrimoniales de la obra puede ser por medio de licencias legales y obligatorias las cuales bajo ciertos parámetros permiten el uso de contenidos (Lipszy c, 1993).

\section{Análisis comparativo de lo "abierto"}

Hasta el momento existen cuatro áreas en donde se desarrollan políticas de acceso abierto (ver cuadro). Estas son: 


\section{Cuadro. Áreas en donde se desarrollan políticas de acceso abierto}

\begin{tabular}{|c|c|c|}
\hline Área & Nombre que adquiere lo abierto & Descripción básica \\
\hline Educación & $\begin{array}{l}\text { Recursos Educativos Abiertos } \\
\text { (REA) - Open Educational } \\
\text { Resources (OER) }\end{array}$ & $\begin{array}{l}\text { Se trata de contenidos abiertos enfocados en la educación. Para que } \\
\text { un recurso sea considerado REA debe ser gratuito, además cumplir } \\
\text { estándares tecnológicos de interoperabilidad y permisos jurídicos } \\
\text { para ser publicados, circulados y descargados en Internet (reproduc- } \\
\text { ción, distribución y comunicación pública) (Unesco, 2009). }\end{array}$ \\
\hline Ciencia & Acceso abierto & $\begin{array}{l}\text { En términos generales, el acceso abierto es la disposición gratuita y } \\
\text { pública en la red que permite a cualquier usuario la lectura, descarga, } \\
\text { copia, distribución, impresión, búsqueda o enlace a textos completos } \\
\text { sin barreras económicas, legales o técnicas. Implica el cumplimiento } \\
\text { de estándares tecnológicos de interoperabilidad y jurídicos de auto- } \\
\text { rizaciones de reproducción, distribución y comunicación pública, me- } \\
\text { nos de modificación (Parada, 2005). }\end{array}$ \\
\hline Gobierno & Gobierno abierto & $\begin{array}{l}\text { Se trata de un conjunto de técnicas tendentes a optimizar la comu- } \\
\text { nicación entre el gobierno y los ciudadanos para lograr un diálogo } \\
\text { dinámico, colaborativo, efectivo y eficaz. Se desarrolla a través de "da- } \\
\text { tos abiertos" estrategia que busca, como ejercicio de transparencia, } \\
\text { compartir sin costo y bajo unos estándares tecnológicos y jurídicos, } \\
\text { datos públicos (Pary cek, Höchtl y Ginner, 2014). }\end{array}$ \\
\hline Cultura & GLAM abierto & $\begin{array}{l}\text { Propone políticas en galerías, biblioteca, archivos y museos (GLAM } \\
\text { por su sigla en inglés). En el sector cultural la tendencia también se } \\
\text { está dando y se centra en estrategias de contenidos y datos abiertos. } \\
\text { Es el más reciente y complejo de definir quizá por su amplio espectro. }\end{array}$ \\
\hline
\end{tabular}

El modelo comparativo de las políticas de acceso abierto en este artículo se centró en las áreas de educación y ciencia que se desarrollan en seis países escogidos por tener avances significativos en leyes, proyectos de leyes, acuerdos y otros recursos legales sobre acceso abierto. Estos países son: España e Inglaterra (una mirada a la Unión Europea), Brasil, Argentina, Australia y Estados Unidos.

La comparación de las áreas de acceso abierto en cada uno de los países se realizó a través de una mirada tecno-céntrica: se parte de la premisa de que los avances tecnológicos facilitan el acceso a la información. Esta visión explica que aún no hay estándares que delimiten de forma clara la manera como se hacen accesibles tecnológica y jurídicamente los datos; sin embargo, el acceso abierto a bienes públi$\cos ^{3}$ es una forma de apropiación y reutilización de conocimiento (Arencibia, 2006).

Dentro del marco legal de cada país sobre cada área de acceso abierto es posible establecer los parámetros sobre difusión y circulación de la producción intelectual que generalmente ha sido financiada por recursos públicos, de igual forma el modelo comparativo permitió reflexionar sobre las medidas que

3 Se adopta la definición de Elinor Ostrom de bien público: "un bien que está disponible a todos y del cual el uso por una persona no sustrae del uso por otros" (1990). Es importante resaltar que precisamente su característica de recursos digitales hace que estos bienes tengan la capacidad de no sustraerse a un solo uso, es esa capacidad de no agotarse la que constituye su abundancia. 
ISSN 0123-1294 | Educ.Educ. Vol. 17. No. 2 | Mayo-Agosto de 2014 | pp. 321-338.

Universidad de La Sabana | Facultad de Educación

han adoptado los Estados para que, dentro de estándares legales, pueda accederse a material protegido.

\section{Ciencia: acceso abierto}

\section{Europa}

Acceso abierto en la investigación: este proyecto fue financiado por El Consejo Europeo de Investigación creado con la finalidad de financiar la investigación dentro de la Comunidad Europea. Los logros más importantes de este plan son la firma de la Declaración sobre Acceso Abierto de la Comisión Europea (2006), los lineamientos de la Comisión al Comité Económico y Social Europeo sobre la información científica en la era digital (2007) y El Libro verde sobre el espacio Europeo de Investigación: nuevas perspectivas (2007).

De acuerdo con estos documentos las publicaciones revisadas por pares de proyectos de investigación financiados por el Consejo deben ser depositados en un repositorio de investigación apropiado como PubMed Central, ArXiv o en un repositorio institucional dentro de los seis meses siguientes a su publicación y serán de acceso abierto. Asimismo, los datos primarios de investigaciones deben ser depositados en las bases de datos pertinentes, preferiblemente de forma inmediata a la publicación en un plazo máximo en seis meses (Consejo Europeo de Lisboa, 2000).

El proyecto piloto de acceso abierto en el séptimo programa marco de investigación y desarrollo: este programa impone condiciones para las subvenciones (presupuesto de 50.500 millones de euros), por ejemplo, que haya un reembolso de hasta un $100 \%$ de los costos totales de las publicaciones en acceso dorado ${ }^{4}$. En el contrato de subvención también se anuncia que los beneficiarios deben asegurarse de que los conocimientos adquiridos se difun-

$4 \quad$ La declaración de Budapest (2002) señala dos rutas para alcanzar el acceso abierto: la ruta dorada que implica la publicación en revistas open access y la ruta verde que hace referencia al depósito de cualquier recurso digital en repositorios institucionales o temáticos. dan tan rápidamente como sea posible y, en caso de no hacerlo, la Comisión está autorizada para difundir estos conocimientos (European Union: European Research, Innovation and Science area, 2009).

En desarrollo de este esquema, desde 2008 se inició el "programa piloto en Acceso Abierto" que obliga a que en determinadas áreas 5 los autores-beneficiarios depositen los resultados en el repositorio institucional o temático correspondiente, adoptando política de acceso abierto en ruta verde que permite un embargo de máximo seis meses. Este programa piloto se monitorea a través del programa OpenAIRE (European Union: European Research, Innovation and Science area, 2011).

La Comisión Europea. Un paso más allá: en julio 2012, la Comisión Europea emitió una recomendación sobre acceso a la información científica, su preservación y reutilización, en la que aboga por el acceso abierto tanto en publicaciones financiadas con dinero público como de las bases de datos de las investigaciones (European Commision: European Research, Innovation and Science area, 2012).

\section{España}

Orden 679 de 2009 de la Comunidad de Madrid: impone la obligación de depositar en acceso abierto las publicaciones derivadas de proyectos de investigación financiados por esa comunidad y cofinanciados por el Fondo Social Europeo, aceptando un embargo de hasta seis y doce meses según el área que corresponda (Abadal, 2012, p. 43). Esta política incluye a las universidades Rey Juan Carlos, Politécnica de Madrid y Carlos III, también a los institutos del Consejo Superior de Investigaciones Científicas ubicados en la Comunidad de Madrid.

Real Decreto 99 de 2011: esta norma regula los doctorados oficiales y establece que una vez se

5 Como energía, medioambiente, salud, tecnologías de la información y las comunicaciones, las infraestructuras de investigación, la ciencia en la sociedad, y las ciencias sociales y humanidades. 
apruebe una tesis doctoral, la universidad se ocupe para que el archivo sea transformado a formato electrónico abierto y se publique en un repositorio institucional (Abadal, 2012).

Ley 14/2011 de Ciencia, tecnología y la innovación: implementa para España el marco normativo que ha desarrollado la Unión Europea, hace extensiva la obligación de depósito en un repositorio institucional o temático de los resultados de investigación que sean financiados "mayoritariamente" con recursos de los presupuestos generales del Estado con un embargo de hasta doce meses. Adicionalmente desarrolla el proyecto Dulcinea que busca identificar y analizar las políticas editoriales de las revistas españolas en relación con el acceso a los textos y su gestión del derecho de autor, para determinar si puede afectar el autoarchivo o depósito en los repositorios (Melero et al., 2012).

\section{Inglaterra}

Declaración de los Consejos de Investigación (2005): es el resultado de una extensa consulta que les permitió establecer una política de recaudación de fondos para la financiación de investigación. Los principios que delimitan esta declaración son: i) las ideas y el conocimiento derivado de la investigación financiada con recursos públicos estará disponible y accesible para uso público tan pronto como fuese posible; ii) los resultados de investigación publicados deberán ajustarse a un control de calidad riguroso a través de la revisión de pares, y iii) los modelos y mecanismos de publicación y acceso a los resultados de investigación deberán ser eficientes en el uso de los recursos públicos (Research Councils UK, 2005).

Anuncio del gobierno inglés sobre mandato a publicaciones científicas (2012): como resultado del informe Accessibility, sustainability, excellence: how to expand access to research publications el gobierno inglés aceptó las recomendaciones dadas por el mismo, también anunció que en el año 2014 todos los estudios científicos subvencionados con recursos públicos estarán disponibles en línea gratuitamente para el uso por parte de universidades, compañías e individuos sin importar en qué lugar del mundo se encuentren. El gobierno también se comprometió a seguir la ruta dorada en el acceso abierto a partir del año 2013 (Finch, 2012).

La política ha sido ampliamente criticada por considerarse que detrás de un aparente apoyo al acceso abierto se está beneficiando el negocio editorial, de modo que Inglaterra gastaría considerables sumas de dinero para mantener el modelo de las editoriales en lugar de apoyar la ruta verde que obliga a cuestionar y replantear ese negocio (Ian, 2012).

\section{Estados Unidos}

Política de Acceso Público NIH: se trata de la política que desde el 2004 desarrolla el National Institute of Health $(\mathrm{NIH})$ por mandato legal. Consiste en una serie de recomendaciones para permitir el acceso gratuito en línea de los artículos resultado de la investigación financiada con sus fondos máximo seis meses después de la publicación en revistas indexadas (Committee Reports of United States Congress, 2005). Inicialmente fue voluntario, en la actualidad es una obligación de quien recibe dichos fondos, en este caso la publicación se hace máximo doce meses después de entregado el documento en PubMed Central.

La política se justifica en la intención de mejorar la capacidad del NIH para preservar los resultados de la investigación y hacerlos más accesibles para los científicos y el público, no exige que la publicación esté asociada con permisos concretos como el de redistribución, ni la sujeción a licencias concretas (National Institute of Health Public Access, 2013).

\section{Argentina}

Sistema Nacional de Repositorios Digitales (SNRD): en el año 2011 se crea el SNRD que impulsa y coordina una red interoperable de repositorios distribuidos físicamente por un sitio web. Este es 
gestionado por instituciones o grupos de instituciones a nivel nacional para aumentar la visibilidad y el impacto de la producción científica y tecnológica de Argentina, por medio del acceso gratuito para leer, descargar, copiar, distribuir, imprimir, buscar y enlazar los textos científicos y usarlos con fines legítimos a la investigación y la educación (Sistema Nacional de Repositorios Digitales, s.f.).

Ley 26899 de 2013 sobre la Creación de Repositorios Digitales Institucionales de Acceso Abierto, Propios o Compartidos: esta iniciativa es presentada ante el Congreso debido a la baja tasa de contenidos registrados en repositorios digitales, por lo que era necesaria una política que estimulara lo abierto en la producción intelectual (Bongiovani y Nakano, 2010). Esta ley asegura la publicación en acceso abierto de aquellas investigaciones cuya actividad ha sido financiada mayoritariamente con los presupuestos generales del Estado, en versión electrónica a partir del Sistema Nacional de Repositorios Digitales (Rosen, 2012).

\section{Australia}

Ley de Conocimiento - Ley OAK (Open Access Knowledge) de 2006: después de un amplio proceso de diseño y discusión, la ley fue aprobada incluyendo informes, guías y publicaciones con la finalidad de crear estrategias para facilitar el acceso ininterrumpido al conocimiento para mejorar los resultados sociales, económicos y culturales de las inversiones del sector púbico en la educación y la investigación (Fitzgerald et al., 2006). La ley prevé un repositorio central digital y un mandato de autoarchivo en repositorios institucionales que incluye disposiciones sobre la gestión del derecho de autor para el libre acceso.

Consejo Nacional de Investigación en Salud y Medicina: desde 2011 los beneficiarios de fondos para investigación de esta institución deben publicar los resultados en acceso libre y gratuito. Se trata de un mandato de autoarchivo en un repositorio de acceso libre, que debe hacerse en un plazo no mayor a un año (Bongiovani, 2009).

\section{Brasil}

Proyectos de ley sobre acceso abierto: el primer proyecto de ley 1120 de 2007 fue archivado y un segundo proyecto de ley 386 de 2011 aún se encuentra en debate. Estas propuestas pretenden permitir el acceso a aquellos documentos resultado de investigaciones realizadas con financiación de recursos públicos (Ortellado, 2009).

Declaración de Salvador (2005), manifiesto brasilero de apoyo al acceso libre (2008): estas recomendaciones propenden por la equidad en el acceso, el dominio de la información y el conocimiento como condición esencial para el desarrollo del país. Varias universidades públicas, como la Universidad de Brasilia, han adoptado sistemas de repositorios abiertos; de igual forma, el Instituto Brasileño de Información en Ciencia y Tecnología (BICT) ha desarrollado un sistema para permitir la visibilidad y el acceso abierto de las revistas que hagan parte del mismo (Portal Brasilero de acceso abierto e información científica, s.f.).

Proyectos SciELO y OASIS: son dos de las más grandes iniciativas de acceso abierto en Brasil que han contado con el apoyo de recursos públicos. La Scientific Electronic Library Online (SciELO) es una biblioteca electrónica fundada por el grupo de apoyo a la investigación del estado de São Paulo que permite la publicación de revistas científicas, y OASIS es el sistema brasilero de biblioteca digital que cuenta con información académica en acceso abierto, es donde se depositan las tesis de las universidades estatales y federales (Guedón, 2011).

\section{Resultados preliminares}

El acceso abierto en ciencia se ha desarrollado en forma muy concreta: se difunden los resultados de investigación en revistas indexadas, de esta forma se establece un estándar que mide la calidad de la investigación y el impacto de la inversión pública en el sector. 
La política pública tiende a expedir mandatos de autoarchivo para el depósito en repositorios institucionales o temáticos que cumplan con estándares tecnológicos para compartir los datos sin que se infrinjan los derechos de autor. De este modo se publica, para consulta en línea y sin costo, a más tardar dentro del año siguiente de finalizada la investigación; normalmente no tienen disposiciones sobre reutilización del material aunque lo faciliten voluntariamente (licenciamiento abierto).

Estos mandatos, cada vez más, provienen de la ley y su alcance puede ser referido a los dineros que destina una organización específica o una línea de investigación para establecer obligaciones de publicación.

Las iniciativas de políticas públicas en ciencia con recursos del Estado son de acceso público, lo que incrementa el número de resultados de investigación y permite a la gran industria ajustarse más fácilmente. Las iniciativas de mayor compromiso con el acceso abierto están en políticas que suelen ser institucionales.

Existe una fuerte tensión entre quienes están a favor del acceso y quienes se inclinan por modelos de producción y difusión soportados en el control del producto, como las industrias farmacéuticas o editoriales. Por este motivo el acceso abierto ha desarrollado un escenario muy concreto que se refiere a las publicaciones en revistas indexadas.

Considerando que hay políticas muy diversas en lo relacionado con acceso abierto y ciencia, es evidente que el éxito está asociado con el nivel de compromiso. El archivo de un proyecto de ley y el congelamiento del otro en Brasil evidencian que sin compromiso político es difícil movilizar una iniciativa legislativa.

La tendencia a desarrollar una política que vincule el mandato a una red de repositorios que permita y monitoree su cumplimiento corresponde a las enseñanzas que han dejado iniciativas pioneras.
Los estudios han demostrado que la tendencia es crear depósitos voluntarios con repositorios y redes para hospedarlos.

\section{Educación: recursos educativos abiertos (REA)}

\section{Europa}

Recursos educativos en la UE: el 24 de mayo de 2000 la Comisión Europea adoptó la Comunicación "e-Learning: concebir la educación del futuro" que recoge como pilar fundamental el desarrollo de servicios y contenidos multimedios de calidad que permitan integrar las TIC en la educación. Para la ejecución de este proyecto la Comisión consideró que es necesario desarrollar y estimular el mercado europeo de los contenidos y servicios que respondan a las necesidades de la comunidad (Comisión e-Learning, 2000).

Esta iniciativa ha propiciado nuevos pronunciamientos de la Comisión y del Consejo de Europa que respaldan los Recursos Educativos Abiertos e instan a los profesores y centros de formación docente a responder a las nuevas demandas de la profesión, por ejemplo, fomentando entornos innovadores de aprendizaje y empleando recursos educativos abiertos (Diario oficial de la Unión Europea, 2008).

Estrategia replantear la educación: La estrategia creada en 2012 por la Comisión Europea propone, entre otras cosas, la integración de la comunicación y la información a través de la tecnología para mejorar el acceso a la educación mediante REA.

En 2013, como resultado de la estrategia, surge la iniciativa Opening Up Education con el objetivo de impulsar la innovación y las aptitudes digitales en los centros educativos. Para consolidar el programa se creó el portal web Open Education Europa en donde se pueden encontrar recursos educativos abiertos de alta calidad producidos en Europa accesibles sin costo y que pueden ser compartidos (Interfblog, 2014). 


\section{España}

Materiales educativos para Internet del Ministerio de Educación, Cultura y Deporte: el Instituto Nacional de Tecnologías Educativas y de Formación del Profesorado premia materiales educativos elaborados para la creación de recursos multimedia de carácter didáctico, accesibles y ajustados a los contenidos curriculares vigentes, las enseñanzas preuniversitarias y susceptibles de ser usados en el aula o en el hogar por parte del profesorado, el alumnado y las familias. Estos materiales son diseñados y puestos en circulación cumpliendo los estándares de los REA (Instituto Nacional de Tecnologías Educativas, 2011).

Repositorio Agrega: es un conjunto de nodos interconectados a través de Internet que configuran un repositorio de recursos digitales educativos que recoge material con licencia Creative Commons y lo clasifica según la posibilidad de reutilización que la licencia otorgue (Fundación Agrega, s.f.). Dentro del repositorio se encuentran los REA producidos por el proyecto del Centro Nacional de Desarrollo Curricular en Sistemas no Propietarios (Cedec) financiado por el Ministerio de Educación, Cultura y Deporte de España y el Índice de recursos educativos abiertos de Andalucía (Mogea), financiado por la Consejería de Educación de la Junta de Andalucía.

\section{Estados Unidos}

Trade Adjustment Assistance Community College and Career Training Grant Program (conocido como TAACCCT): en enero de 2011 los departamentos de Trabajo y de Educación crearon un fondo de financiación para materiales educativos con características de REA para cursos de capacitación en "community colleges" los cuales son una estrategia de educación institucional. Los materiales que se produzcan deberán ser públicos, cumplir con los estándares de interoperabilidad y estar licenciados con Creative Commons Atribución 3.0 (US deparment of Labor, s.f.).

La polémica de este proyecto radica en la posibilidad de que las editoriales y empresas del sector educativo tomen estos productos y los comercialicen permitiendo otros modelos de negocio de pago paralelos al de acceso digital abierto.

\section{Argentina}

Portal educativo de la Nación Argentina: esta propuesta del Ministerio de educación busca articular las políticas de educación y tecnologías de Argentina dentro de una filosofía de compartir. Hasta el momento no hay un proyecto concreto sobre el desarrollo de material con estándares de REA (Estado Argentino, s.f.).

\section{Australia}

National Digital Learning Resources Network: proyecto del Consejo Ministerial de Asuntos para el Desarrollo Temprano de Educación para la Niñez y Juventud, administrado por Education Services Australia $(E S A)^{6}$. En el marco de esta iniciativa se administra y extiende la colección nacional de recursos digitales que se busca alinear con el currículo australiano que apoya intercambios de los recursos entre las diferentes jurisdicciones.

Es importante señalar que los recursos deben mantener los licenciamientos y manejos de derecho de autor en la colección nacional, la infraestructura técnica y el hospedaje de los sistemas para que faciliten la distribución nacional de estos (Consulado de Australia, s.f.)

\section{Brasil}

Ley de recursos educativos abiertos: esta iniciativa, sancionada en 2012, dispone que los recursos educativos financiados con dineros del gobierno federal sean publicados con licencias abiertas para favorecer el acceso. Aclara que los recursos producidos por los funcionarios públicos en desarrollo de sus funciones deben ser abiertos o publicados en el 6 Fundación de propiedad de todos los ministerios de educa-
ción del país. 
marco del acceso abierto, a su vez promueve el apoyo del gobierno para sistemas federados en la distribución y archivo de estos recursos (Vollme, 2011).

Recursos educativos financiados por la Secretaría de Educación de São Paulo: la Secretaría Municipal de Educación de la ciudad de São Paulo adoptó la licencia Creative Commons AtribuciónNoComercial-Compartirlgual (BY-NC-SA) para todo su material educativo y didáctico. Se trata de una disposición o mandato que se hace efectivo a través de la divulgación de los recursos que se publiquen en su portal por Internet (Prefeitura da Cidade de São Paulo, s.f.).

\section{Resultados preliminares}

A diferencia del área en ciencia, el modelo de desarrollo de los materiales didácticos es bastante variado pues buena parte de los recursos provienen del sector público pero la necesidad de ajustarlos a lo local y casi individual permite proponer más modelos descentralizados y diversos.

La tendencia en política pública se ha acentuado más en proyectos de cooperación entre instituciones para compartir recursos educativos. Son pocos por ahora los mandatos legales que buscan que la inversión de dinero público tenga como resultado material público.

Las redes de repositorios han surgido en esquemas de cooperación pública y privada con énfasis en la necesidad de reutilización del material.

Para que los recursos educativos puedan reutilizarse de manera óptima los mandatos deben ser más cuidadosos y diversos, pues deberán considerar claramente la población y el objetivo de cada proyecto.

Hay diversas iniciativas de redes de repositorios apoyadas desde el sector público y dispuestas a acoger los recursos educativos abiertos; sin embargo, adquieren vida y lógica cuando van asociados con mandatos.
La política pública en esta materia parece tener más éxito cuando está vinculada en forma íntima con comunidades específicas, por esta razón existen más decisiones del Ejecutivo para poner a disposición dineros públicos a fin de apoyar proyectos de REA que generen obligaciones a los beneficiarios.

Aún no existe un modelo de política pública claro ya que la diversidad de productos didácticos es mayor que en la ciencia lo que dificulta reglas claras respecto al tema.

La mayoría de los proyectos son de inversión y cooperación en que el Estado propone mecanismos de cooperación entre las instituciones que proveen al sector educativo y participa apoyando a las redes de repositorios que los hospeden.

\section{Conclusiones}

Abierto en Europa: el sector público europeo es un gran impulsor de las ideas de lo abierto, es el continente que quizás más proyectos ha desarrollado dentro de la última década sobre el tema.

Dada la diversidad de países, la naturaleza jurídica de los modelos de acceso abierto es variada: en algunos casos se promulgan actos legislativos nacionales aislados, como es el caso de los REA, o políticas regionales que formulan estructuras impositivas en los países de la Unión Europea sobre todo en la promulgación de resultados científicos.

En cuanto al alcance de los proyectos se evidencia una imposición de condiciones sobre los recursos públicos, como también un gran apoyo estatal a la financiación de proyectos en los sectores de la ciencia y la educación.

Se observa un compromiso general por el acceso público más que por el acceso abierto. Aunque algunas disposiciones delimitan parámetros y estándares de lo abierto como en el caso de los REA, lo cierto es que en los proyectos en ciencia aún existen trabas para lograr estándares de reutilización de la información. 
Abierto en Estados Unidos: la tendencia de lo abierto en el sector público ha estado sujeta a discusiones que se han materializado en normas de diferentes niveles. El alcance de los proyectos implementados pretende, a partir de recursos o bienes públicos, potenciar la inversión en ciencia y educación.

Se palpa un compromiso general por el acceso público con una mayor conciencia entre las diferencias entre lo público y lo abierto que en otras regiones. Resulta interesante la injerencia que tienen la sociedad civil y los grupos académicos en la propuesta, implementación y administración de modelos de acceso abierto en Estados Unidos.

Abierto en Argentina: el sector público argentino ha avanzado en una ruta legislativa para impulsar las ideas de lo abierto en el campo de la ciencia, en este sentido son pioneros en la región.

En cuanto al alcance de los proyectos en ciencia existe una imposición de condiciones sobre los recursos públicos lo que evidencia una recopilación de las experiencias europeas y estadounidenses; en los REA aún no hay políticas claras.

Abierto en Australia: como en los casos anteriores la naturaleza jurídica de los actos que implementan las políticas es diversa, en Australia se viene avanzando en lo abierto a través de informes y múltiples proyectos.

En cuanto al alcance de los proyectos, en todos los sectores el eje central es la imposición de condiciones sobre material producido con dineros públicos. Es importante resaltar que las iniciativas legislativas en educación van más allá de la declaración de apertura, lo que propone un esquema de acceso abierto mas no de acceso público.

Abierto en Brasil: es posible identificar que el apoyo público al acceso abierto ha adquirido especial significado a través de disposiciones legales, como es el caso de los REA, en donde a diferencia de otros países se regula a través de leyes. Los proyectos evidencian una apuesta directa por el acceso abierto, pues se están adoptando licencias abiertas.

Las iniciativas propuestas se justifican en la realidad de las comunidades sociales que son las movilizadoras de esta tendencia; sin embargo, dado el poco compromiso político del gobierno algunas de estas no han podido ser implementadas.

\section{Reflexiones finales}

El acceso abierto ha nacido de la relevancia que tiene la masificación y facilitación de la información por medio de formatos digitales para abordar y solucionar problemas sociales, como el acceso a la educación y el conocimiento. Las leyes y políticas públicas al respecto estipulan la manera como las restricciones legales y de control tecnológico deben ser abordadas para que no se conviertan en un obstáculo para acceder abiertamente a contenidos científicos y educativos.

Una de las medidas adoptadas por las políticas de acceso abierto es la utilización de licencias o autorizaciones generales como Creative Commons que permitan un uso más libre de los contenidos y la redistribución de los mismos, a diferencia del acceso público que implica modelos de autorización menos amplios.

El nacimiento de proyectos y la incorporación de políticas públicas sobre acceso abierto en educación y ciencia no ha sido similar en los países de estudio, lo que pone de manifiesto que aún existen grandes retos para la consolidación y el mantenimiento de un sistema mundial de acceso abierto que logre conectar sin ninguna clase de barrera a toda la comunidad interesada en utilizar contenidos bien sea por ruta verde o dorada.

Entre los retos se encuentra la formación de investigadores para que entiendan cómo funcionan los repositorios institucionales y el autoarchivo de material protegido por leyes de derecho de autor. En los REA se necesita unificar requisitos y estándares 
en las iniciativas para lograr que los recursos puedan ser utilizados en otras poblaciones diferentes a las que se dirige el proyecto por el que se creó. En el caso de la ciencia es necesario instaurar estrategias de mercado que permitan sobrevivir a las editoriales y centros de investigación pero que a su vez admitan la publicación de obras y resultados de investigación bajo el concepto de "abierto".

\section{Referencias}

Abadal, E. (2012). Acceso abierto a la Ciencia. Barcelona: Editorial UOC, Colección El profesional de la información.

Arencibia, J. (2006). Las iniciativas para el acceso abierto a la información científica en el contexto de la Web Semántica. Biblios: Revista electrónica de bibliotecología, archivología y museología, 2 (25-26), 1-14.

Bongiovani, P.y Nakano, S. (2010). El Sistema Nacional de Repositorios Digitales (SNRD). La experiencia de articulación y coordinación institucional de los repositorios digitales en ciencia y tecnología. En Jornada Virtual del Acceso Abierto Argentina 2010 (pp. 1-12). Buenos Aires: e- Colabora.

Bongiovani, P. (2009). Traducción Kit de politicas de acceso abierto de Eloy Rodrigues. Buenos Aires: ACCAP.

Committee Reports of United States Congress (2005). House Report 108-636-Departments of Labor, Health and Human Services, and Education, and related agencies appropriation bill. Washington: The Library of Congress.

Concha, G. y Naser, A. (2012). El desafío hacia el gobierno abierto en la hora de la igualdad. Santiago de Chile: Comisión Económica para América Latina y el Caribe (Cepal).

Craveiro, G., Machado, J. y Ortellado, P. (2008). El mercado de libros técnicos y científicos en Brasil. São Paulo: Canal 6 Projetos Editoriais.

Diario Oficial de la Unión Europea (2008). Conclusiones del Consejo y de los Representantes de los Estados miembros, reunidos en el seno del Consejo, de 22 de mayo de 2008, sobre el fomento de la creatividad y la innovación en la educación y la formación. 2008/C 141/10. Bruselas: Consejo Europeo.

European Union: European Research, Innovation and Science area (2012). Survey on open access in FP7. Luxembourg: Publications Office of the European Union.

European Union: European Research, Innovation and Science area (2011). National Open Acces and Preservation Policies in Europe. Luxembourg: Publications Office of the European Union.

European Commision: European Research, Innovation and Science area (2009). The Gender Challenget in research funding : Assessing the European national scenes. Luxembourg: Office for Official Publications of the European Communities.

Finch, J. (2012). Accessibility, sustainability, excellence: how to expand access to research publications. Working Group on Expanding Access to Published Research Findings. London: Research Information Network. 
ISSN 0123-1294 | Educ.Educ. Vol. 17. No. 2 | Mayo-Agosto de 2014 | pp. 321-338.

Universidad de La Sabana | Facultad de Educación

Fitzgerald, B., Fitzgerald, A., Perry, M., Kiel-Chisholm, S., Driscoll, E., Thampapillai, D. et al. (2006). Creating a Legal Framework for Copyright Management of Open Access within the Australian Academic and. Brisbane: OAK Law Report Number 1.

Guédon, J. (2011). El acceso abierto y la división entre ciencia "principal” y “periférica”. Revista crítica y emancipación, 3 (6), 135-180.

Hargreaves, I. (2011). A review of Intellectual Property and Growth. United Kingdoms: UK government.

Hogge, B. (2010). Open Data Study. United Kingdom: Open Society Foundations.

Hoosen, S. (2012). Survey on Governments'open educational Resources (OER) Policies. Paris: Unesco.

Ian, S. (2012). Free access to British scientific research within two years. London: The Guardian.

Iglesias, C. y Labastida, I. (2006). Guía sobre gestión de derechos de autor y acceso abierto a bibliotecas, servicios de documentación y archivos. Madrid: Asociación Española de Documentación e Información.

Landes, W. \& Posner, R. (2003). The structure of intellectual property law. Massachusett: The Belknap Press.

Lipszy c, D. (1993). Derecho de autory derechos conexos. Cerlalc: Ediciones Unesco.

Melero, R., Abad, M., Abadal E., Benito, C., Giménez, F., Rodríguez, G. y Rodríguez N. (2012). Dulcinea: iniciativa para el análisis de los derechos de copyright y autoarchivo de revistas científicas españolas. En XI Jornadas Españolas de Documentación de mayo de 2009 (pp. 117-122). Zaragoza: Fesabid.

Ortellado, P. (2009). Acceso abierto en Brasil. São Paulo: GPOPAI.

Ostrom, E. (1990). Governing the Commons: The Evolution of Institutions for Collective Action. Cambridge: Cambridge University Press.

Parada, A. (2005). El acceso abierto (open access) y el futuro de la edición en el ámbito biomédico: una figura con múltiples aristas. El profesional de la información, 14 (5), 326-334.

Pary cek, P., Höchtl, J. \& Ginner, M. (2014). Open government Data implementation evaluation. Journal of Theoretical and applied electronic commerce research, 9 (2), 80-99.

Plata López, L. (2010). Responsabilidad civil por infracciones al derecho de autor. Barranquilla: Grupo editorial Ibañez - Ediciones Uninorte.

Unesco (2009). Open Educational Resources: Conversations in Cyberspace. París: Unesco.

Unesco (2013). Informe de la consulta Latinoamericana y del Caribe sobre acceso abierto a información e investigación científica. París: Unesco.

Vollme, T. (2011). Brazil introduces OER into federal legislation and adopts local government policy. Recuperado el 23 de noviembre de 2012 de: http://creativecommons.org/weblog/entry/27698 


\section{Páginas de Internet:}

Comisión de información de Australia (s.f.). Draft report: Engage Getting on with Government 2.o; Submission to the Government 2.0 Taskforce. Recuperado el 12 de noviembre de 2012 de: http://www.oaic.gov.au/privacy/privacy-archive/privacy-submissions-archive/draft-report-engage-getting-on-with-government20-submission-to-the-government-20-taskforce-december-2009

Departamento de industria del gobierno australeano (s.f.). NIS Report. Recuperado el 7 de octubre de 2012 de: http://www.innovation.gov.au/Innovation/Policy/Documents/NISReport.rtf

Rosen, C. (2012). Argentina avanza hacia una ley de acceso abierto. Recuperado el 20 de octubre de 2012 de: http://www.scidev.net/es/science-communication/open-access/news/argentina-avanza-hacia-una-leyde-acceso-abierto.html

National Institute of Health Public Acces (s.f.). US. Recuperado el 17 de octubre de 2012 de: http://publicaccess. nih.gov/FAQ.htm\#814

House of Commons (2004). Scientific publications: free for all? Recuperado el 18 de octubre de 2012 de: http:// www.publications.parliament.uk/pa/cm200304/cmselect/cmsctech/399/399.pdf

Research Councils UK (s.f.). Councils UK' updated position statement on access to research outputs. Recuperado el 9 de septiembre de 2012 de: http://www.rcuk.ac.uk/RCUK-prod/assets/documents/ documents/2005statement.pdf

Sistema Nacional de Repositorios Digitales (s.f.). Adhesión al Sistema. Recuperado el 22 de noviembre de 2012 de: http://repositorios.mincyt.gob.ar/adhesion.php

Portal Brasilero de acceso abierto e información científica (s.f.). Sobre o oasisbr. Recuperado el 22 de novimbre de 2012 de: http://oasisbr.ibict.br/sobre-o-oasisbr

Comisión e-Learning (2000). Comunicación - Concebir la educación del futuro. Recuperado el 22 de noviembre de 2012 de: http://eur-lex.europa.eu/LexUriServ/LexUriServ.do?uri=CELEX:52000DCo318:ES:HTML

Interfblog (2000). Recursos educativos abiertos para la enseñanza y puesta en común de recursos a través de cinco cursos en línea para la formación de profesores. Recuperado el 10 de junio de 2012 de: http://blog. educalab.es/intef/2014/03/o4/recursos-educativos-abiertos-para-la-ensenanza-busqueda-creacion-ypuesta-en-comun-de-recursos-a-traves-de-cinco-cursos-en-linea-para-la-formacion-de-profesores/

Instituto Nacional de Tecnologías Educativas y de Formación del Profesorado (2011). Premios a materiales educativos. Recuperado el 4 de julio de 2012 de: http://www.ite.educacion.es/es/premios-a-materiales-educativos/convocatoria-2011

Fundación Agrega (s.f.). Recuperado el 12 de julio de 2012 de: http://agrega.educacion.es/visualizadorcontenidos/Faqs/Faqs.do\#anchor1 
ISSN $0123-1294$ | Educ.Educ. Vol. 17. No. 2 | Mayo-Agosto de 2014 | pp. 321-338.

Universidad de La Sabana | Facultad de Educación

US Deparment of Labor (s.f.). Trade Adjustment Assistance Community College and Career Training Grant Program, program summary. Recuperado el 22 de noviembre de 2012 de: http://www.doleta.gov/taaccct/

Estado Argentino (s.f.). Portal educativo del Estado Argentino. Recuperado el 29 de septiembre de 2012 de: http://www.educ.ar/

Consulado de Australia (s.f.). Education Services Australia consultada el 20 de agosto de 2012. Recuperado el 9 de septimbre de 2012 de: http://www.esa.edu.au/about-us/governance

Prefeitura da Cidade de São Paulo (s.f.). Portal da Prefeitura da Cidade de São Paulo. Recuperado el 17 de octubre de 2012 de: http://www.portalsme.prefeitura.sp.gov.br/default.aspx

\section{Declaraciones, tratados y leyes:}

Declaración de Salvador, 23 de septiembre de 2005 en Brasil.

Declaración de Bethesda, 11 de abril de 2003.

Declaración de Berlín, 6 de octubre de 2003.

Declaración de Principios de la Cumbre Mundial sobre la Sociedad de la información, 8 de diciembre de 2003.

Declaración sobre Acceso Abierto de la Comisión Europea, 28 de abril de 2006.

El Libro verde sobre el espacio Europeo de Investigación: nuevas perspectivas, abril 5 de 2007.

Iniciativa de Acceso abierto o Declaración de Budapest, 1 de marzo de 2002.

Ley de Conocimiento - Ley OAK en Australia, 1 de agosto de 2006.

Ley 14 de 1 de junio 2011 de Ciencia, Tecnología y la Innovación en España.

Ley 26899 del 13 de noviembre de 2013 sobre la Creación de Repositorios Digitales Institucionales de Acceso Abierto, Propios o Compartidos en Argentina.

Lineamientos de la Comisión al Parlamento Europeo, al Consejo y al Comité Económico y Social Europeo sobre la información científica en la era digital: acceso, difusión y preservación, 2 de febrero de 2007.

Manifiesto Brasilero de apoyo al acceso libre en Brasil, 8 de junio de 2008

Orden 679 de 19 de febrero de 2009 de la Comunidad de Madrid.

Real Decreto de España 99, 28 de enero de 2011.

Sesión especial del Consejo Europeo de Lisboa, conclusiones de la presidencia. Lisboa, 24 de marzo de 2000. 